\title{
Mathematische Leitfäden
}

Herausgegeben von

em. o. Prof. Dr. phil. Dr. h.c. mult. G. Köthe, Universität Frankfurt/M., und o. Prof. Dr. rer. nat. G. Trautmann, Universität Kaiserslautern

\section{Locally Convex Spaces}

by Dr. phil. Hans Jarchow

Professor at the University of Zürich 
Prof. Dr. phil. Hans Jarchow

Born 1941 in Bremerhaven. Studies in Hamburg and Zürich from 1960 to 1966. Received diploma in 1966 and Ph.D. in 1967 from the University of Zürich. At the University of Zürich, lecturer from 1969 to 1970, assistant professor from 1970 to 1979 , and associate professor beginning in 1979. Visiting professor at the University of Maryland from 1974 to 1975.

CIP-Kurztitelaufnahme der Deutschen Bibliothek

\section{Jarchow, Hans:}

Locally convex spaces / by Hans Jarchow. -

Stuttgart : Teubner, 1981.

(Mathematische Leitfäden)

ISBN 978-3-322-90561-1 ISBN 978-3-322-90559-8 (eBook)

DOI 10.1007/978-3-322-90559-8

This work is subject to copyright. All rights are reserved, whether the whole or part of the material is concerned, specifically those of translation, reprinting, re-use of illustrations, broadcasting, reproductions by photocopying machine or similar means, and storage in data banks.

Under $\S 54$ of the German Copyright Law where copies are made for other than private use, a fee is payable to the publisher, the amount of the fee to be determined by agreement with the publisher.

(C) B. G. Teubner, Stuttgart 1981

Setting: Schmitt u. Köhler, Würzburg

Cover design: W. Koch, Sindelfingen 
To Szazy 


\section{Preface}

The present book grew out of several courses which I have taught at the University of Zürich and at the University of Maryland during the past seven years. It is primarily intended to be a systematic text on locally convex spaces at the level of a student who has some familiarity with general topology and basic measure theory. However, since much of the material is of fairly recent origin and partly appears here for the first time in a book, and also since some well-known material has been given a not so well-known treatment, I hope that this book might prove useful even to more advanced readers. And in addition I hope that the selection of material marks a sufficient set-off from the treatments in e.g. N. Bourbaki [4], [5], R.E. Edwards [1], K. Floret-J. Wloka [1], H.G. Garnir-M. De Wilde-J.Schmets [1], A. Grothendieck [13], H. Heuser [1], J. Horváth [1], J.L. Kelley-I. Namioka et al. [1], G. Köthe [7], [10], A.P. RobertsonW. Robertson [1], W. Rudin [2], H.H. Schaefer [1], F. Trèves [1], A. Wilansky [1].

A few sentences should be said about the organization of the book. It consists of 21 chapters which are grouped into three parts. Each chapter splits into several sections. Chapters, sections, and the statements therein are enumerated in consecutive fashion. Cross references are usually k.m.n. meaning that reference is made to statement $\mathrm{n}$ of section $m$ in chapter $k$. Inside of $k . m$ (i.e. of section $m$ in chapter $k$ ) reference is simply to $\mathrm{n}$ rather than to k.m.n. Definitions are not listed as separate statements, they are only distinguished from the text by using spaced type characters. The end of a proof is marked by

Starting from Chapter 2, each chapter concludes with a reference section, intended to give an (admittedly vague) impression on the origin of several results presented in the foregoing sections, to provide furtheron a certain amount of additional information, and also to suggest further reading. It should be mentioned, however, that despite its extent, and even with regard to only those topics which are covered in this book, the corresponding bibliography is far from being complete.

Let us continue by looking very briefly at the content of the book. The first part, consisting of chapters $1-7$, is devoted to the elementary theory of general topological vector spaces. Chapter 1 presents a certain amount of basic material on linear spaces of arbitrary dimension, Chapter 2 is concerned with the most fundamental concepts on topological vector spaces, Chapter 3 discusses various completeness concepts, and Chapter 4 deals with inductive linear topologies, in particular with quotients, direct sums, and inductive limits. One of the most important principles, the closed graph 
theorem, is treated together with its relatives in Chapter 5 from a rather general point of view, within the setting of the webbed spaces of De Wilde and Robertson.

Locally convex spaces make their first appearance in Chapter 6. Their elementary theory is even developed within the frame of locally $r$-convex spaces, $0<r \leqslant 1$. Chapter 7 then already lives from these concepts. The Hahn-Banach theorem is proved together with its most important consequences, a version of the Krein-Milman theorem for locally $r$-convex spaces is presented, and the Riesz representation theorem is derived for finite regular Borel measures on an arbitrary completely regular space. The validity of the Hahn-Banach theorem is what makes locally convex spaces superior to other classes of topological vector spaces. It assures the existence of sufficiently many continuous linear forms to support a powerful duality theory. This theory is the central theme of the second part of the book. Its fundamental aspects are discussed in Chapter 8. In Chapter 9, the influence of considering the equicontinuous sets in the dual of a locally convex space as an inductive family of compact spaces is examined. Among others, Grothendieck's completeness theorem, the BanachDieudonné theorem, and Pták's results on B-completeness are derived, and the theorems of Eberlein, Krein, and S̆mulian are proved in substantial generality.

Chapter 10 presents an introduction to the theory of Schwartz spaces through local (or Mackey) convergence, which does not follow the known tracks; instead, it seems to be more effective than the usual approaches. Further topics are diametral dimensions and power series spaces. In Chapter 11 some additional important classes of locally convex spaces are investigated, such as barrelled spaces, quasi-barrelled spaces, reflexive and semi-reflexive spaces, Montel and semi-Montel spaces. The basic material on uniformly convex Banach spaces and on Hilbert spaces is included for the purpose of later reference and in order to be self-contained.

The unifying theme of Chapter 12 is dual characterization of complete metrizability for locally convex spaces, and related questions. It is based on Grothendieck's theory of $D F$-spaces, but it extends this theory by inclusion of several recent results on certain weakenings of barrelledness, on fundamental sequences of bounded sets, and by clarifying the relationship to Schwartz topologies. Chapter 13 is devoted to a fairly general development of the theory of bornological and ultrabornological spaces. Among others, it contains the theorems of Mackey-Ulam and of Nachbin-Shirota with full proofs.

Topological bases and related objects are investigated in some detail in Chapter 14 . Among the topics treated in that chapter are the continuity theorem, the weak basis theorem, shrinking and boundedly complete bases, the Orlicz-Pettis theorem, unconditional bases, and absolute bases.

The final part of the book is concerned with tensor products of locally convex spaces, various spaces of operators, and related classes of locally convex spaces, in particular with nuclear locally convex spaces. Tensor products make their natural appearance when trying to obtain results on bilinear mappings by using results on linear mappings. The tensor product in the category of locally convex spaces is Grothendieck's projective tensor product which is investigated in Chapter 15. In a sense dual to this is 
the notion of the injective tensor product of locally convex spaces which is discussed in Chapter 16.

Chapter 17 is devoted to some classical spaces of continuous operators between locally convex spaces, in particular between Banach spaces, and their relations to tensor products. Chapter 18 comprises the basic results on spaces with approximation property. The theory of ideals of operators in Banach spaces, in the sense of Pietsch, is worked out in some detail in Chapter 19. Some particular aspects of this theory in presence of Hilbert spaces are discussed in Chapter 20, by using Grothendieck's inequality. The study of ideals leads to a rather rapid development of the theory of nuclear spaces in Chapter 21. Besides giving various characterizations of such spaces e.g. through ideals, tensor products, summability conditions, diametral dimensions, Choquet simplexes, special attention is paid to co-nuclear spaces, strongly nuclear spaces, and to Schauder bases in nuclear spaces.

In the preceding lines I have tried to give some rough information on what is contained in this book. Much more could be said about what is not contained in it. Some specific omissions may even be considered serious by other specialists. I am surely aware of this. But I was unfortunately not quite free concerning the extent of this book, and furtheron it was not my intention to write an encyclopedic treatise on the subject.

It was already mentioned that a prerequisite for a successful reading of the book is familiarity with the basic facts from general topology (without uniform spaces) and measure theory. These facts will not be rederived here. More than what will be needed is contained in N. Bourbaki [2], J. Dugundji [1], J.L. Kelley [1], or H. Schubert [1] (topology), and in J.J. Benedetto [1], P.R.Halmos [1], or W.Rudin [1] (measure theory). Convergence in topological spaces will be described by filters (N. Bourbaki [2]) or by nets (J.L. Kelley [1]), according to whatever seems to be more convenient. If a reader feels uncertain about some set-theoretic manipulations, then he is recommended to read P.R. Halmos [2].

The necessary backgound on general linear spaces will be prepared in the first chapter; this will also provide an opportunity to fix some notation. Otherwise, the notation and terminology employed here will be standard and need no extra explanation. The symbols $\mathbb{N}, \mathbb{Z}, \mathbb{Q}$ will always be used to denote the sets of all natural numbers (= positive integers), of all integers, and of all rational numbers, respectively. The symbol $\mathbb{K}$ is reserved to denote either the field $\mathbb{R}$ of real numbers, or the field $\mathbb{C}$ of complex numbers. In addition, $\mathbb{N}_{0}, \mathbb{R}_{+}, \mathbb{R}_{*}$ will be used to denote the respective sets $\mathbb{N} \cup\{0\},\{r \in \mathbb{R} \mid r \geqslant 0\}$, and $\{r \in \mathbb{R} \mid r>0\}$. Occasionally, it will be convenient to call "rational numbers" not only the elements of $\mathbb{Q}$ but also those of $\mathbb{Q} \times \mathbb{Q} \subset \mathbb{C}$.

In preparation of the present book, I have received valuable suggestions from many colleagues. It is a great pleasure to record my gratitude to them at this place. In particular, I am grateful to G. Köthe, R. Ott, and K.D. Schmidt for reading parts of the manuscript and for their helpful comments, to E. Dubinsky, A. Pietsch, and W. Ruess for several fruitful discussions, to M. A. Kaashoeck for a stimulating idea, and to W.L. Hill and E.W. Straeuli for linguistic assistance. I am also indebted to the publishers for their care and cooperation. 


\section{Preface}

Finally, my thanks go to Jutta for encouragement and patience during the writing of this book, and to Janka and Thomas for their lively taking part in preparing the final version of the manuscript.

Zürich, Spring 1980

H. Jarchow 


\section{Contents}

\section{Part I: Linear Topologies}

\section{Vector Spaces}

1.1 Generalities. . . . . . . . . . . . . . . . . . 15

1.2 Elementary Constructions . . . . . . . . . . . . . . . 16

1.3 Linear Maps . . . . . . . . . . . . . . . . . . . . . . . . . . 17

1.4 Linear Independence. . . . . . . . . . . . . . . . . . . . . 19

1.5 Linear Forms. . . . . . . . . . . . . . . . . . . . 20

1.6 Bilinear Maps and Tensor Products . . . . . . . . . . . . . 22

1.7 Some Examples . . . . . . . . . . . . . . . . . . . 25

\section{Topological Vector Spaces}

2.1 Generalities. . . . . . . . . . . . . . . . . 30

2.2 Circled and Absorbent Sets . . . . . . . . . . . . . . . 32

2.3 Bounded Sets. Continuous Linear Forms . . . . . . . . . . . 34

2.4 Projective Topologies . . . . . . . . . . . . . . . . 35

2.5 A Universal Characterization of Products . . . . . . . . . . 36

2.6 Projective Limits . . . . . . . . . . . . . . . . . . . . . . . . . 37

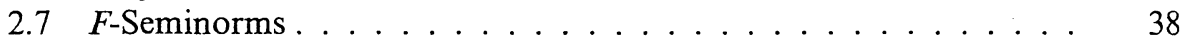

2.8 Metrizable Tvs . . . . . . . . . . . . . . . . . . 40

2.9 Projective Representation of Tvs . . . . . . . . . . . . . . 42

2.10 Linear Topologies on Function and Sequence Spaces. . . . . . . 43

2.11 References . . . . . . . . . . . . . . . . . 54

\section{Completeness}

3.1 Some General Concepts . . . . . . . . . . . . . . . . . . . . 56

3.2 Some Completeness Concepts. . . . . . . . . . . . . . . . . . 57

3.3 Completion of a Tvs. . . . . . . . . . . . . . 59

3.4 Extension of Uniformly Continuous Maps . . . . . . . . . 61

3.5 Precompact Sets . . . . . . . . . . . . . . . . . . . 64

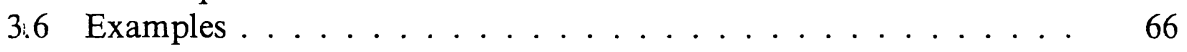

3.7 References . . . . . . . . . . . . . . . . 73 


\section{Inductive Linear Topologies}

4.1 Generalities. . . . . . . . . . . . . . . . . . . . 74

4.2 Quotients of Trs . . . . . . . . . . . . . . 76

4.3 Direct Sums . . . . . . . . . . . . . . . . . . . 78

4.4 Some Completeness Results . . . . . . . . . . . . . . . . . . . . . . 80

4.5 Inductive Limits . . . . . . . . . . . . . . . . . . . . . . 82

4.6 Strict Inductive Limits . . . . . . . . . . . . . . . . . . . . 84

4.7 References . . . . . . . . . . . . . . . . 86

5 Baire Trs and Webbed Trs

5.1 Baire Category . . . . . . . . . . . . . . . . . 87

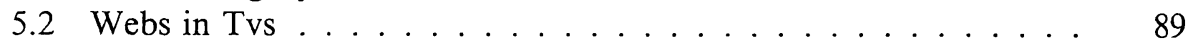

5.3 Stability Properties of Webbed Tvs. . . . . . . . . . . . . . 90

5.4 The Closed Graph Theorem . . . . . . . . . . . . . . . . . . . . . . . . . . 92

5.5 Some Consequences . . . . . . . . . . . . . . . . . . . . . . . . . 95

5.6 Strictly Webbed Tvs . . . . . . . . . . . . . . . . . . . . . . . . . . . . 96

5.7 Some Examples . . . . . . . . . . . . . . . . . . . . . . . . 97

5.8 References . . . . . . . . . . . . . . . . . 99

6 Locally $r$-Convex Tvs

$6.1 r$-Convex Sets. . . . . . . . . . . . . . . . . . 101

$6.2 r$-Convex Sets in Tvs. . . . . . . . . . . . . . 103

6.3 Gauge Functionals and $r$-Seminorms. . . . . . . . . . . . . . 104

6.4 Continuity Properties of Gauge Functionals. . . . . . . . . . . . 106

6.5 Definition and Basic Properties of $\mathrm{Lc}_{r} \mathrm{~s} \ldots \ldots . . . . . . . . .108$

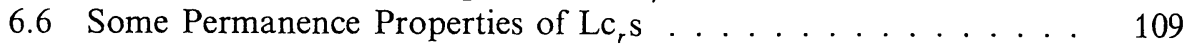

6.7 Bounded, Precompact, and Compact Sets . . . . . . . . . . . . . 112

6.8 Locally Bounded Tvs . . . . . . . . . . . . . . . . . . . . . . . 114

6.9 Linear Mappings Between $r$-Normable Tvs . . . . . . . . . . . . 117

6.10 Examples . . . . . . . . . . . . . . . . . . . . . . 119

6.11 References . . . . . . . . . . . . . . . . . . . . 124

7 Theorems of Hahn-Banach, Krein-Milman, and Riesz

7.1 Sublinear Functionals . . . . . . . . . . . . . . . . . . 125

7.2 Extension Theorem for Lcs . . . . . . . . . . . . . . . . . . 127

7.3 Separation Theorems . . . . . . . . . . . . . . . . 130

7.4 Extension Theorems for Normed Spaces. . . . . . . . . . . . . . 132

7.5 The Krein-Milman Theorem . . . . . . . . . . . . . . . . . . . . . 133

7.6 The Riesz Representation Theorem. . . . . . . . . . . . . . . . 137

7.7 References . . . . . . . . . . . . . . . . . . 144 


\section{Part II: Duality Theory for Locally Convex Spaces}

\section{Basic Duality Theory}

8.1 Dual Pairings and Weak Topologies . . . . . . . . . . . . . 145

8.2 Polarization . . . . . . . . . . . . . . . . . . . . . 148

8.3 Barrels and Disks . . . . . . . . . . . . . . . . . . 150

8.4 Bornologies and $\mathscr{B}$-Topologies . . . . . . . . . . . . . 152

8.5 Equicontinuous Sets and Compactologies . . . . . . . . . . . 156

8.6 Continuity of Linear Maps . . . . . . . . . . . . . . . . . . . . 160

8.7 Duality of Subspaces and Quotients . . . . . . . . . . . . 163

8.8 Duality of Products and Direct Sums . . . . . . . . . . . . . . 165

8.9 The Stone-Weierstrass Theorem . . . . . . . . . . . . . . . . 169

8.10 References . . . . . . . . . . . . . . . . . . 173

9 Continuous Convergence and Related Topologies

9.1 Continuous Convergence . . . . . . . . . . . . . . . . . . . . 174

9.2 Grothendieck's Completeness Theorem . . . . . . . . . . . . . . 176

9.3 The Topologies $\gamma^{t}$ and $\gamma \ldots \ldots \ldots \ldots \ldots$. . . . . . . . . . . . . . . . . . . . 18

9.4 The Banach-Dieudonné Theorem. . . . . . . . . . . . . . . . 181

$9.5 B$-Completeness and Related Properties . . . . . . . . . . . . . 183

9.6 Open and Nearly Open Mappings . . . . . . . . . . . . . . . . 184

9.7 Application to $B$-Completeness . . . . . . . . . . . . . . . . . . 186

9.8 On Weak Compactness . . . . . . . . . . . . . . . . . . . . . . . . 189

9.9 References . . . . . . . . . . . . . . . . . 193

10 Local Convergence and Schwartz Spaces

$10.1 \not B$-Convergence. Local Convergence. . . . . . . . . . . . . . . 195

10.2 Local Completeness . . . . . . . . . . . . . . . . . . . . . 197

10.3 Equicontinuous Convergence. The Topologies $\eta^{t}$ and $\eta$. . . . . 199

10.4 Schwartz Topologies . . . . . . . . . . . . . . . . . . 201

10.5 A Universal Schwartz Space . . . . . . . . . . . . . . . . . 204

10.6 Diametral Dimension. Power Series Spaces . . . . . . . . . . . 207

10.7 Quasi-Normable Lcs . . . . . . . . . . . . . . . . . . . . . . . . 214

10.8 Application to Continuous Function Spaces . . . . . . . . . . 216

10.9 References . . . . . . . . . . . . . . . . . . . 217

\section{Barrelledness and Reflexivity}

11.1 Barrelled Lcs . . . . . . . . . . . . . . . . . . . . . 219

11.2 Quasi-Barrelled Lcs. . . . . . . . . . . . . . . . . . . . . . 222 
11.3 Some Permanence Properties . . . . . . . . . . . . . . . 223

11.4 Semi-Reflexive and Reflexive Lcs . . . . . . . . . . . . . . . 227

11.5 Semi-Montel and Montel Spaces . . . . . . . . . . . . . . . 229

11.6 On Fréchet-Montel Spaces. . . . . . . . . . . . . . . . . . 231

11.7 Application to Continuous Function Spaces . . . . . . . . . . 233

11.8 On Uniformly Convex Banach Spaces . . . . . . . . . . . . 236

11.9 On Hilbert Spaces . . . . . . . . . . . . . . . . . . . . . . . . 241

11.10 References . . . . . . . . . . . . . . . . . 247

\section{Sequential Barrelledness}

$12.1 \ell_{\infty}$-Barrelled and $c_{0}$-Barrelled Lcs . . . . . . . . . . . . 249

$12.2 \aleph_{0}$-Barrelled Lcs . . . . . . . . . . . . . . . . . . 251

12.3 Absorbent and Bornivorous Sequences . . . . . . . . . . . 253

$12.4 \quad D F$-Spaces, $g D F$-Spaces, and $d f$-Spaces . . . . . . . . . . . 257

12.5 Relations to Schwartz Topologies . . . . . . . . . . . . 263

12.6 Application to Continuous Function Spaces . . . . . . . . . . 266

12.7 References. . . . . . . . . . . . . . . . . . 269

\section{Bornological and Utrabornological Spaces}

13.1 Generalities . . . . . . . . . . . . . . . . . 271

$13.2 \mathscr{B}$-Convergent and Rapidly $\mathscr{B}$-Convergent Sequences . . . . . . 273

13.3 Associated Bornological and Ultrabornological Spaces . . . . . . 276

13.4 On the Topology $\beta\left(E^{\prime}, E\right)^{\text {bor } \ldots \ldots . . . . . . . . . . . . . ~ . ~ . ~} 279$

13.5 Permanence Properties. . . . . . . . . . . . . . . . . 281

13.6 Application to Continuous Function Spaces . . . . . . . . . . 283

13.7 References . . . . . . . . . . . . . . . . . 288

\section{On Topological Bases}

14.1 Biorthogonal Sequences . . . . . . . . . . . . . . . . . . . . 289

14.2 Bases and Schauder Bases . . . . . . . . . . . . . . . . 292

14.3 Weak Bases. Equicontinuous Bases . . . . . . . . . . . . . 295

14.4 Examples and Additional Remarks . . . . . . . . . . . . . . 299

14.5 Shrinking and Boundedly Complete Bases . . . . . . . . . . . . 302

14.6 On Summable Sequences. . . . . . . . . . . . . . . . . . 305

14.7 Unconditional and Absolute Bases . . . . . . . . . . . . . . 309

14.8 Orthonormal Bases in Hilbert Spaces. . . . . . . . . . . . 315

14.9 References...................... 320 


\section{Part III Tensor Products and Nuclearity}

\section{The Projective Tensor Product}

15.1 Generalities on Projective Tensor Products . . . . . . . . . . . . 323

15.2 Tensor Product and Linear Mappings . . . . . . . . . . . . . . . 326

15.3 Linear Mappings with Values in a Dual . . . . . . . . . . . . . 329

15.4 Projective Limits and Projective Tensor Products . . . . . . . . . . 331

15.5 Inductive Limits and Projective Tensor Products . . . . . . . . . 333

15.6 Some Stability Properties . . . . . . . . . . . . . . . . . 335

15.7 Projective Tensor Products with $\mathscr{L}_{1}(\mu)$-spaces. . . . . . . . . 338

15.8 References. . . . . . . . . . . . . . . . . . . . 341

\section{The Injective Tensor Product}

$16.1 \varepsilon$-Products and $\varepsilon$-Tensor Products. . . . . . . . . . . . . . . 343

16.2 Tensor Product and Linear Mappings . . . . . . . . . . . . . . . . 347

16.3 Projective and Inductive Limits . . . . . . . . . . . . . . . . . . 350

16.4 Some Stability Properties . . . . . . . . . . . . . . . . . . 353

16.5 Spaces of Summable Sequences . . . . . . . . . . . . . . . 357

16.6 Continuous Vector Valued Functions. . . . . . . . . . . . . . . . 360

16.7 Holomorphic Vector Valued Functions. . . . . . . . . . . . . . 362

16.8 References . . . . . . . . . . . . . . . . . . . . . 366

\section{Some Classes of Operators}

17.1 Compact Operators. . . . . . . . . . . . . . . . . . 368

17.2 Weakly Compact Operators . . . . . . . . . . . . . . . 372

17.3 Nuclear Operators . . . . . . . . . . . . . . . . . . . . . . . 376

17.4 Integral Operators . . . . . . . . . . . . . . . . . . . . . . . 380

17.5 The Trace for Finite Operators . . . . . . . . . . . . . . . . . 386

17.6 Some Particular Cases . . . . . . . . . . . . . . . . . . . . 391

17.7 References...................... 395

\section{The Approximation Property}

18.1 Generalities . . . . . . . . . . . . . . . . . . . . 397

18.2 Some Stability Properties . . . . . . . . . . . . . . . . 401

18.3 The Approximation Property for Banach Spaces . . . . . . . . . 403

18.4 The Metric Approximation Property . . . . . . . . . . . . . . . 408

18.5 The Approximation Property for Concrete Spaces. . . . . . . . . 410

18.6 References. . . . . . . . . . . . . . . . . 417 
19 Ideals of Operators in Banach Spaces

19.1 Generalities . . . . . . . . . . . . . . . . . . . 418

19.2 Dual, Injective, and Surjective Ideals . . . . . . . . . . . . . . 420

19.3 Ideal-Quasinorms. . . . . . . . . . . . . . . . . . . . . . 422

$19.4 t_{p}$-Sequences. . . . . . . . . . . . . . . . . . . . . 425

19.5 Absolutely $p$-Summing Operators . . . . . . . . . . . . . . 428

19.6 Factorization . . . . . . . . . . . . . . . . . 431

19.7 p-Nuclear Operators . . . . . . . . . . . . . . . . . 434

19.8 p-Approximable Operators. . . . . . . . . . . . . . . . . 439

19.9 Strongly Nuclear Operators . . . . . . . . . . . . . . . . . . . . . . . . 443

19.10 Some Multiplication Theorems . . . . . . . . . . . . . . . . 445

19.11 References. . . . . . . . . . . . . . . . . . . . . 449

20 Components of Ideals on Particular Spaces

20.1 Compact Operators on Hilbert Spaces . . . . . . . . . . . . . 451

20.2 The Schatten-von Neumann Classes . . . . . . . . . . . . . 453

20.3 Grothendieck's Inequality . . . . . . . . . . . . . . . . . . . . 458

20.4 Applications. . . . . . . . . . . . . . . . . . . . . 462

$20.5 \mathscr{P}_{p}$ and $\mathscr{N}_{q}$ on Hilbert Spaces . . . . . . . . . . . . 467

20.6 Composition of Absolutely Summing Operators . . . . . . . . . 470

20.7 Weakly Compact Operators on $\mathscr{C}(K)$-Spaces . . . . . . . . . . 472

20.8 References. . . . . . . . . . . . . . . 476

\section{Nuclear Locally Convex Spaces}

21.1 Locally Convex $\mathscr{A}$-Spaces . . . . . . . . . . . . . . . . . . . . 478

21.2 Generalities on Nuclear Spaces . . . . . . . . . . . . . . . 482

21.3 Further Characterizations by Tensor Products. . . . . . . . . . . 486

21.4 Nuclear Spaces and Choquet Simplexes . . . . . . . . . . . . . 489

21.5 On Co-Nuclear Spaces . . . . . . . . . . . . . . . . . . . . 491

21.6 Examples of Nuclear Spaces . . . . . . . . . . . . . . . . . . 496

21.7 A Universal Generator . . . . . . . . . . . . . . . . . . . 500

21.8 Strongly Nuclear Spaces . . . . . . . . . . . . . . . . . 504

21.9 Associated Topologies . . . . . . . . . . . . . . . . . . . . . 508

21.10 Bases in Nuclear Spaces . . . . . . . . . . . . . . . . 510

21.11 References...................... 517

Bibliography . . . . . . . . . . . . . . . . . 520

List of Symbols . . . . . . . . . . . . . . . . . . . . . . . 541

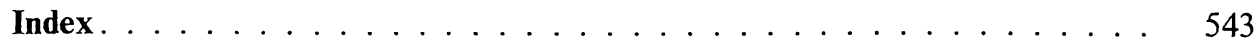

